\title{
Oncogenic ETS proteins mimic activated RAS/MAPK signaling in prostate cells
}

\author{
Peter C. Hollenhorst, ${ }^{1,6}$ Mary W. Ferris, ${ }^{1}$ Megan A. Hull, ${ }^{1}$ Heejoon Chae, ${ }^{2}$ Sun Kim, ${ }^{2,5}$ \\ and Barbara J. Graves ${ }^{3,4}$ \\ ${ }^{1}$ Medical Sciences, Indiana University School of Medicine, Bloomington, Indiana 47405, USA; ${ }^{2}$ Bioinformatics Program, School \\ of Informatics and Computing, Indiana University, Bloomington, Indiana 47405, USA; ${ }^{3}$ Department of Oncological Sciences, \\ Huntsman Cancer Institute, University of Utah, Salt Lake City, Utah 84112, USA; ${ }^{4}$ Howard Hughes Medical Institute, Chevy \\ Chase, Maryland 20815, USA
}

The aberrant expression of an oncogenic ETS transcription factor is implicated in the progression of the majority of prostate cancers, $40 \%$ of melanomas, and most cases of gastrointestinal stromal tumor and Ewing's sarcoma. Chromosomal rearrangements in prostate cancer result in overexpression of any one of four ETS transcription factors. How these four oncogenic ETS genes differ from the numerous other ETS genes expressed in normal prostate and contribute to tumor progression is not understood. We report that these oncogenic ETS proteins, but not other ETS factors, enhance prostate cell migration. Genome-wide binding analysis matched this specific biological function to occupancy of a unique set of genomic sites highlighted by the presence of ETS- and AP1-binding sequences. ETS/AP-1-binding sequences are prototypical RAS-responsive elements, but oncogenic ETS proteins activated a RAS/MAPK transcriptional program in the absence of MAPK activation. Thus, overexpression of oncogenic ETS proteins can replace RAS/MAPK pathway activation in prostate cells. The genomic description of this ETS/AP-1-regulated, RAS-responsive, gene expression program provides a resource for understanding the role of these ETS factors in both an oncogenic setting and the developmental processes where these genes normally function.

[Keywords: prostate cancer; ETS; ChIP-seq; RAS/MAPK; cell migration]

Supplemental material is available for this article.

Received July 25, 2011; revised version accepted September 12, 2011.

In cancer cells, aberrant gene expression programs result from alterations in the signaling pathways that regulate transcription factor function, or from the mutation or altered expression of transcription factors themselves. Deciphering the role of a transcription factor requires understanding how these proteins are targeted to specific genomic binding sites, how they influence transcription once bound, and how these functions are modified by signaling pathways. However, overlapping functions among the thousands of transcription factors encoded by the human genome has made it difficult to assign specific oncogenic mechanisms.

The ETS family of transcription factors exemplifies this specificity problem (Hollenhorst et al. 2011a). The 28 human ETS proteins bind DNA via a conserved ETS DNAbinding domain and recognize similar DNA sequences. All ETS proteins bind sites with the core sequence GGA

${ }^{5}$ Present address: School of Computer Science and Engineering, Seoul National University, 599 Kwangak-ro, Gwanak-Gu, Seoul 151-742, Korea. ${ }^{6}$ Corresponding author.

E-mail pchollen@indiana.edu.

Article is online at http://www.genesdev.org/cgi/doi/10.1101/gad.17546311. and most bind with highest affinity to the extended consensus CCGGAAGT (Wei et al. 2010). This lack of intrinsic DNA sequence specificity is contrasted by unique biological functions for each ETS family member (Hollenhorst et al. 2011a). We showed previously that genomic targets of ETS transcription factors can include two distinct classes (Hollenhorst et al. 2007, 2009). First are the "redundant" binding sites found in the proximal promoters of housekeeping genes. Binding sites in this class are characterized by the consensus ETS sequence (CCGGAAGT) and thus have the potential to bind any ETS protein with relatively high affinity. Second are the "specific" binding sites that are found more often in enhancer regions associated with genes that mediate the specific biological functions of an ETS family member. Specific target sites are characterized by a lower-affinity ETS sequence, often AGGAA, and are sometimes flanked by binding sites for other transcription factors. This is consistent with a model that low-affinity ETS-binding sites, supported by cooperative interactions with neighboring transcription factors, mediate specific ETS functions.

A limited number of ETS transcription factors have been shown to be oncogenic in humans. Normal human tissues 
coexpress the majority of the ETS family genes (Hollenhorst et al. 2004). Yet, some tumors and cancer cell lines express high levels of an additional ETS gene that is either absent or at low levels in the normal tissue. For example, chromosomal rearrangements result in overexpression of full-length or truncated versions of the ETS genes ERG, ETV1, ETV4, or ETV5 in $50 \%-70 \%$ of prostate cancers, with the most common being the TMPRSS2-ERG rearrangement (Tomlins et al. 2005, 2006; Helgeson et al. 2008). Furthermore, $>40 \%$ of melanomas and most gastrointestinal stromal tumors (GISTs) express high levels of ETV1 (Chi et al. 2010; Jane-Valbuena et al. 2010). ERG, $E T V 1$, and ETV5 overexpression increases invasiveness of prostate cell lines (Cai et al. 2007; Tomlins et al. 2007, 2008; Helgeson et al. 2008). ERG overexpression promotes prostate tumor progression from prostatic intraepithelial neoplasia (PIN) to early invasion stage in mouse models in combination with $\mathrm{PI} 3 \mathrm{~K} / \mathrm{AKT}$ pathway activation or androgen receptor (AR) overexpression (Carver et al. 2009;
King et al. 2009; Zong et al. 2009). Thus, a subset of ETS genes that includes ERG, ETV1, ETV4, and ETV5 has an oncogenic function. However, the mechanism that differentiates these oncogenic ETS family members from other ETS family members is not clear.

Phylogenetic comparison of ETS domain sequences indicates that ETS genes altered in prostate cancer cluster into two ETS family subclasses: ERG and PEA3 (Fig. 1A). ETV1, ETV4, and ETV5 are closely related and comprise the PEA3 subfamily. Members of this subfamily have sequence similarity that extends the length of the proteins. However, the only sequence conservation between ERG and the PEA3 subfamily is in the ETS DNA-binding domain. FLI1 and FEV from the ERG subfamily have not been found to be overexpressed in prostate cancer. It is not known whether ERG has the same or a distinct role in prostate cancer compared with ETV1, ETV4, and ETV5. The subset of ETS genes implicated in prostate cancer could reflect the relative likelihood of chromosomal rearrange-
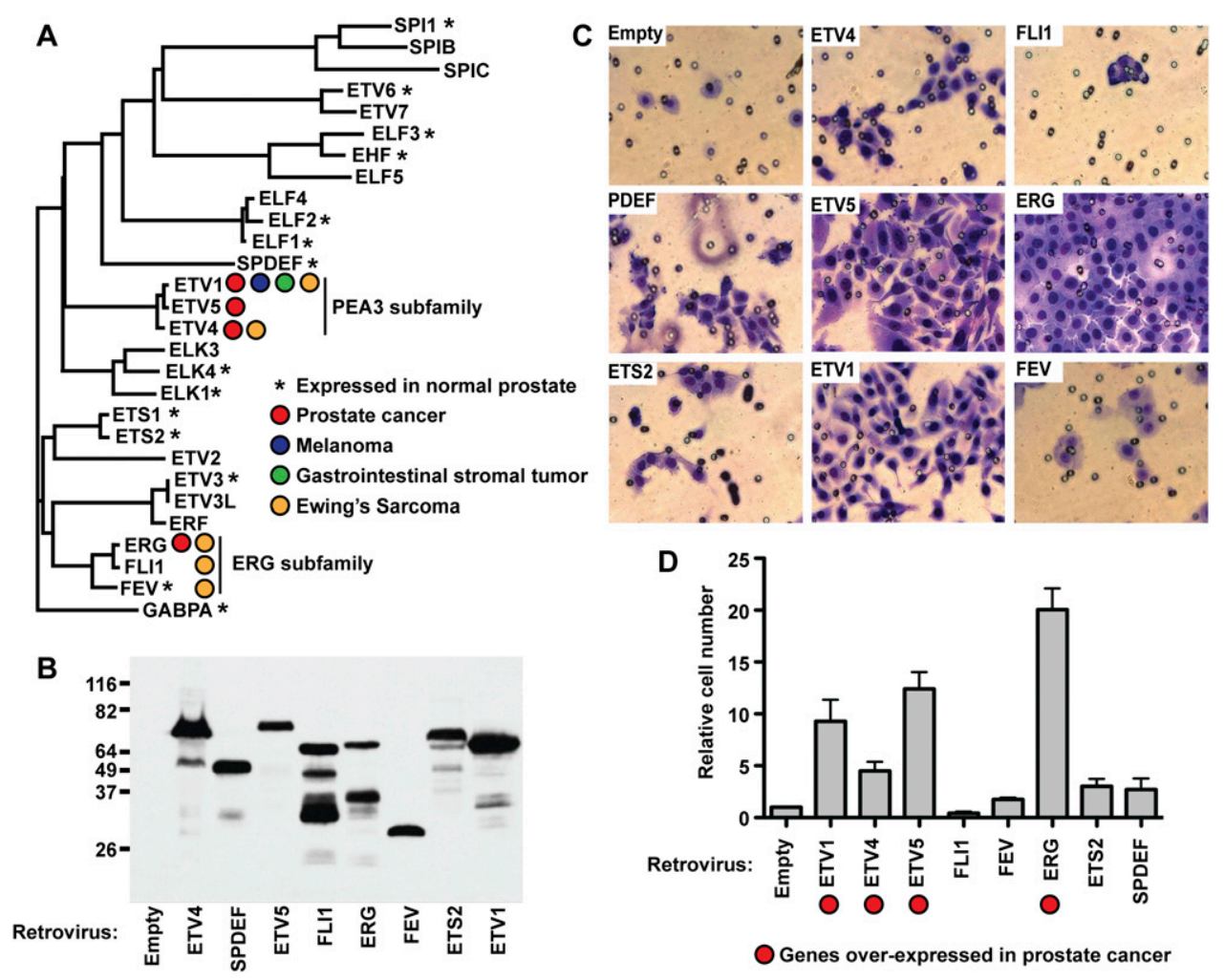

Figure 1. A subset of ETS proteins can increase prostate cell migration. (A) A phylogram tree of human ETS domain sequences identifies subfamilies of one to three members each. ERG and PEA3 subfamilies are labeled. ETS family members expressed in normal prostate (>10 mRNA copies per cell) or overexpressed in prostate cancer, melanoma, or GIST are indicated. Also indicated are family members involved in EWS-ETS fusions in Ewing's sarcoma. (B) A protein immunoblot with anti-Flag antibody of whole-cell extracts from RWPE-1 cells expressing the indicated ETS gene or empty vector from an integrated retroviral vector. Molecular weight markers (kilodaltons) are shown on the left. Predicted molecular weights, including Flag, are ETV4, $57 \mathrm{kDa}$; SPDEF, 40 kDa; ETV5, $61 \mathrm{kDa}$; FLI1, $54 \mathrm{kDa}$; ERG, $57 \mathrm{kDa}$; FEV, $28 \mathrm{kDa}$; ETS2, $56 \mathrm{kDa}$; and ETV1, $58 \mathrm{kDa}$. Higher apparent molecular weights are consistent with previous reports (Wu and Janknecht 2002; Baert et al. 2007; Hollenhorst et al. 2011b). (C) RWPE-1 cells expressing the indicated ETS gene were cultured in a Boyden chamber with $8-\mu \mathrm{m}$ pores in medium lacking growth supplements and allowed to migrate toward medium containing supplements. Cells that migrated out of the chamber were stained and a representative experiment is shown. $(D)$ Migrating cells from $C$ were counted and are reported relative to the number of migrating empty vector RWPE-1 cells. Cell number is the mean and SEM of four biological replicates, each consisting of the mean of two technical replicates. Genes found in chromosomal translocations in prostate cancer are marked. 
ment (Lin et al. 2009; Mani et al. 2009) and/or a common oncogenic function. In the latter case, it is not clear how the oncogenic function of these four ETS genes might differ from the nononcogenic function of ETS transcription factors expressed in normal prostate. Of particular interest, the two most highly expressed ETS genes in normal prostate, EHF and SPDEF, have predicted roles as prostate cancer tumor suppressors (Gu et al. 2007; Cangemi et al. 2008; Turner et al. 2011).

The RAS/RAF/MEK/ERK (RAS/MAPK) signaling pathway is often activated in cancer. Prominent examples include the KRAS mutation found in $80 \%$ of pancreatic cancers (Bos 1989) and missense mutations of BRAF associated with $66 \%$ of malignant melanomas (Davies et al. 2002). ETS family members, including ETS1, ETS2, ELK1, ELK3, ELK4, GABPA, SPIB, ETV1, ETV4, and ETV5, can be phosphorylated by MAPKs, resulting in increased transcriptional activation (Charlot et al. 2010). However, ETSbinding sequences are reported to act as RAS-responsive elements only in certain contexts, such as in juxtaposition to binding sequences for the AP-1 or SRF transcription factors (Wasylyk et al. 1998). Furthermore, the identity of the ETS proteins that occupy these sites in vivo is not clear. The importance of RAS/MAPK signaling to cancer suggests a link to the oncogenic nature of ERG, ETV1, ETV4, and ETV5. However, because ETS proteins expressed in normal prostate (ETS1, ETS2, ELK1, ELK3, ELK4, and GABPA/ can also respond to this pathway, it is not known how this represents a specific oncogenic pathway.

Here we test the specificity of oncogenic and nononcogenic ETS transcription factors in prostate cell migration, and monitor genome-wide occupancy and activation of RAS/MAPK target genes. ERG, ETV1, ETV4, and ETV5, but not other ETS genes, increased cellular migration, indicating a specific oncogenic mechanism mediated by these four family members. Genome-wide location analysis revealed that the oncogenic ETS transcription factors have a specific binding pattern that is distinct from nononcogenic ETS proteins. Furthermore, this specific binding is closely correlated with the presence of a binding site for the AP-1 class of transcription factors. Oncogenic ETS proteins activated a MEK/ERK-regulated gene expression program in the absence of ERK activation. These data support a model in which oncogenic ETS proteins can promote a RAS/MAPK transcriptional program in cancer cells that lack an activating mutation in this pathway.

\section{Results}

A cell migration role for ERG, ETV1, ETV4, and ETV5, but not other ETS factors

ETS factors are implicated in the stage of prostate oncogenesis that transitions from hyperplasia to early invasive carcinoma (Shen and Abate-Shen 2010). Thus, we propose cell migration to be a surrogate marker for the role of ETS factors in prostate carcinogenesis. To compare the function of multiple ETS genes in the same cell line, we measured the effect of overexpression on cellular migration in the RWPE-1 cell line. The RWPE-1 cell line is derived from normal prostate, is untransformed, and does not overexpress any of the ETS genes implicated in prostate cancer (Bello et al. 1997; Hollenhorst et al. 2011b). However, like normal prostate tissue, RWPE-1 cells express other ETS members (Fig. 1A). Retroviral transduction created stable cell lines expressing Flag-tagged versions of each of the four ETS genes overexpressed in prostate cancer $(E R G$, ETV1, ETV4, and ETV5) or four ETS genes not overexpressed in prostate cancer. This latter set includes the two additional members of the ERG subfamily, FLI1 and $F E V$; the prostate tumor suppressor SPDEF; and ETS2. This retroviral expression system resulted in similar levels of full-length, tagged ETS proteins (Fig. 1B). The role of each ETS gene in cellular migration was tested in a Boyden chamber (transwell) assay. RWPE-1 cells showed very little migration in the absence of an overexpressed ETS gene (Fig. 1C). ERG, the ETS gene most commonly overexpressed in prostate cancer, induced the highest level of migration in RWPE-1 cells. In all, the four ETS genes overexpressed in prostate cancer induced higher levels of migration than the four nononcogenic ETS genes (Fig. 1D). Thus, the ETS genes that are overexpressed in prostate cancer have a role in cell migration that is distinct from other ETS family members, including the two closest ERG homologs, FLI1 and FEV.

\section{Oncogenic ETS proteins co-occupy a specific class} of genomic targets

A unique oncogenic function for ERG, ETV1, ETV4, and ETV5 suggests that these ETS proteins have a distinct set of transcriptional targets that differ from other ETS family members. To identify these targets, the genomic occupancy of oncogenic and nononcogenic ETS proteins was mapped in RWPE-1 cells using chromatin immunoprecipitation (ChIP) coupled with next-generation sequencing (ChIP-seq). Occupied regions were identified as those with more sequencing reads in the ChIP sample than the input sample in a sliding window at a false discovery rate (FDR) of $<0.01$, and a subset was confirmed by quantitative PCR (qPCR) (Supplemental Fig. S1A). Antibodies to the endogenous ETS proteins ETS1 and GABPA identified 2180 and 8070 bound genomic regions, respectively (Supplemental Table S1). Flag antibody ChIP-seq from RWPE1 cells expressing Flag-ETV1 or Flag-ERG was compared in one analysis with input DNA sequencing and in a second analysis with Flag antibody ChIP-seq from RWPE-1 cells without a Flag-tagged protein. Peaks were only considered as bound regions if they had an FDR of $<0.01$ in both analyses. By this method, ETV1 had 8736 bound regions and ERG had 2314 bound regions (Supplemental Table S1). ERG-bound regions showed high overlap with ERG-bound regions from a previous study (Supplemental Fig. S1B). The ETV1-bound regions overlapped with ERGbound regions significantly $(P<0.0001$ by Fisher's exact test) more often than with regions occupied by either of the nononcogenic ETS proteins, ETS1 or GABPA (Fig. 2A). Therefore, despite belonging to diverse ETS subclasses, ERG and ETV1 shared a common set of target genes when expressed in the same cell line. 


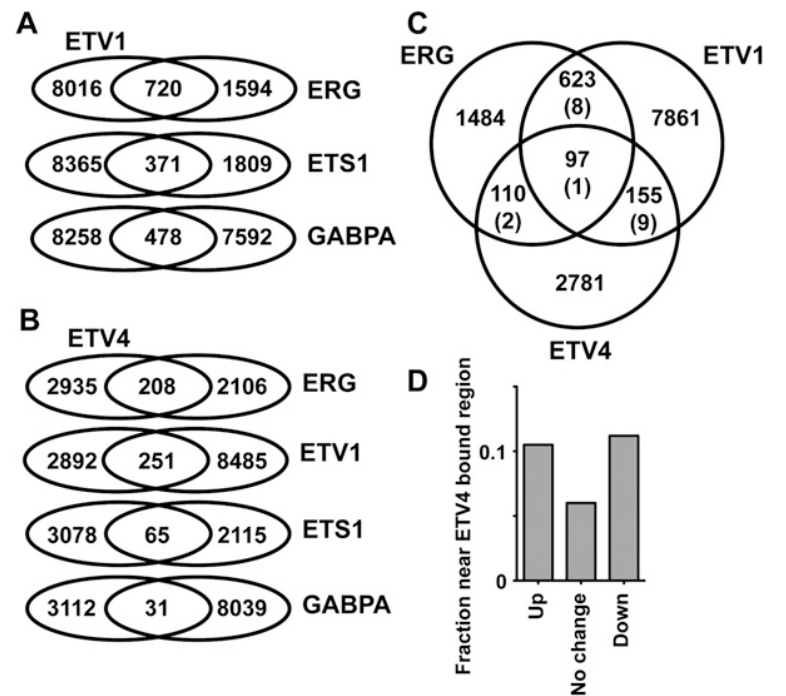

Figure 2. Oncogenic ETS proteins occupy a common set of genomic regions. Diagrams illustrate the number of bound regions identified by ChIP-seq for each ETS protein. Bound regions were considered overlapping if any genomic coordinate was shared. (A) Overlaps from RWPE-1 ChIP-seq. ETS1 and GABPA ChIP-assayed endogenous proteins. ETV1 and ERG ChIP-assayed retrovirally expressed Flag-tagged proteins. $(B)$ Overlaps between endogenous ETV4 in PC3 cells and ETS proteins in RWPE-1 cells from $A$. $(C)$ Overlaps between ETV4 in PC3 cells, and Flag-ETV1 or Flag-ERG in RWPE-1 cells. Numbers in parentheses represent random predictions reported as the mean overlap in 100 iterations of randomly generated size- and GC content-matched genomic regions. Note that a smaller overlap between PC3 and RWPE-1 cell results is likely due to cell line differences. $(D)$ Fraction of genes either up $(542$ genes with a mean expression increase greater than twofold), down (508 genes with a mean expression decrease greater than twofold), or unchanged in an ETV4 shRNA knockdown in PC3 cells (Hollenhorst et al. 2011b) that are nearest (distance to TSS) to an ETV4-bound region.

The overlap of ETV1 and ERG targets could represent a common biological function, or simply be due to the experimental design, which compared exogenous with endogenous ETS factors. To control for this possibility, we interrogated endogenous ETV4-bound regions in PC3 cells by ChIP-seq with an ETV4 antibody. We showed previously that PC3 cells overexpress ETV4 and no other oncogenic ETS factor. Furthermore, ETV4 expression promotes PC3 cell migration and is essential for growth in soft agar, indicating that this cell line provides a cancer context for the genomic analysis (Hollenhorst et al. 2011b). ETV4 had 3143 bound regions. ETV4-bound regions overlapped with regions bound by ETV1 and ERG at a higher frequency than regions bound by ETS1 or GABPA (Fig. 2B). A comparison of all three oncogenic ETS target lists indicated a level of overlap up to 97-fold higher than predicted by comparison with randomly generated, size-matched lists (Fig. 2C). In conclusion, even in diverse cell lines and assayed by different experimental systems, oncogenic ETS proteins bind a common set of genomic targets that is distinct from that bound by nononcogenic ETS proteins.
Oncogenic ETS protein targets are consistent with a specific role in prostate cancer progression

Our previous work indicates that ETS transcription factors can have a redundant function in the proximal promoters of housekeeping genes or more specific functions in enhancer regions (Hollenhorst et al. 2009). Regions occupied by ERG (66\%), ETV1 (78\%), and ETV4 (96\%) were located in regions distal (>500 base pairs [bp]) from transcription start sites (TSSs), consistent with a specific function. Potential gene targets for the regions with overlapping occupancy of ERG, ETV1, and ETV4 were identified by assignment to the nearest TSS. This gene list was searched for overrepresented functional categories using the GoMiner program (Zeeberg et al. 2003). The highestranking categories were consistent with roles in organismal development, cell proliferation, and blood vessel morphogenesis (Table 1), all categories that could connect with the migration behavior analyzed in Figure 1. These categories are similar to those previously identified for genes upregulated by ETV1 or ETV4 overexpression in RWPE-1 cells (Tomlins et al. 2007; Hollenhorst et al. 2011b) and match the normal biological role of ERG in vasculogenesis (McLaughlin et al. 2001; Ellett et al. 2009). In PC3 cells, genes changing expression after depletion of ETV4 by shRNA targeting were enriched for nearby ETV4-bound regions, indicating direct regulation (Fig. 2D). These findings indicate that regions bound by oncogenic ETS proteins regulate a specific gene expression program.

\section{Unique DNA sequences associate with oncogenic ETS occupancy}

ERG, ETV1, and ETV4 co-occupied a group of genomic sites that are distinct from those occupied by other ETS proteins, indicating a mechanism of genomic recruitment that applies only to this subset of proteins. To identify genomic DNA sequences that might mediate this preferential recruitment, regions bound by both oncogenic and nononcogenic ETS transcription factors were subjected to an unbiased search for overrepresented DNA sequence motifs using the MEME algorithm (Fig. 3A; Bailey and

Table 1. Functional categories of genes near ERG-, ETV1-, and ETV4-occupied regions

\begin{tabular}{ll}
\hline Category $^{\mathrm{a}}$ & $P$-value \\
\hline Cell differentiation & $5 \times 10^{-9}$ \\
Multicellular organismal development & $2 \times 10^{-7}$ \\
Blood vessel development & $2 \times 10^{-6}$ \\
Response to organic substance & $4 \times 10^{-6}$ \\
Cell communication & $4 \times 10^{-6}$ \\
Growth & $4 \times 10^{-6}$ \\
Blood vessel morphogenesis & $5 \times 10^{-6}$ \\
Cell proliferation & $1 \times 10^{-6}$ \\
Signal transduction & $1 \times 10^{-5}$ \\
Angiogenesis & $1 \times 10^{-5}$
\end{tabular}

${ }^{\mathrm{a}}$ Regions with overlapping occupancy of ERG, ETV1, and ETV4 (97) were mapped to the nearest RefSeq gene and gene lists were analyzed by GoMiner. Overrepresented categories are listed in the order returned.

${ }^{\mathrm{b}} P$-value for each category from GoMiner. 

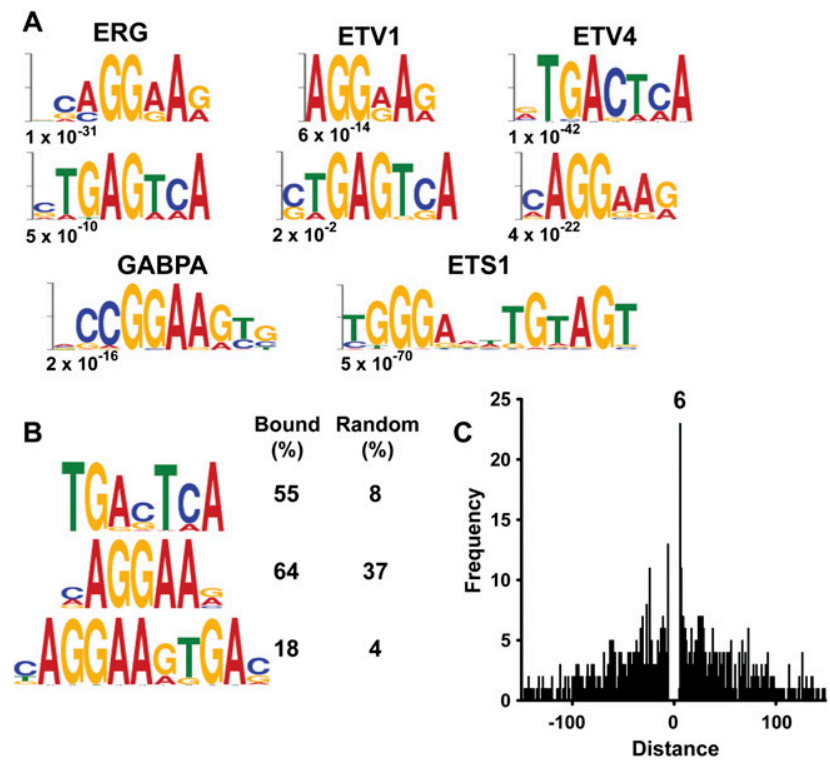

Figure 3. Genomic regions occupied by oncogenic ETS proteins have similar sequence motifs. $(A)$ Regions occupied by the indicated ETS proteins were searched for overrepresented sequence motifs by MEME. The most enriched motifs are shown in logo form, where letter height corresponds to frequency. The $E$, or expect-value returned by MEME, is shown below each sequence. (B) Representative motifs from the 97 regions occupied commonly by ERG, ETV1, and ETV4 are shown. The percentage of these regions with the indicated motif is shown (Bound). "Random" indicates the percentage of an equally sized set of randomly selected genomic regions containing the same motif. $(C)$ Spacing of sequence motifs found in regions occupied by ERG. The distance from all AGGAA sequences in regions bound by ERG to the nearest TGANTCA sequence was recorded, and the frequency of distances between -150 and +150 was plotted as a histogram. Distance was counted from the first nucleotide of each sequence. The most frequent position was +6 , corresponding to the sequence AGGAANTGANTCA.

Elkan 1994). The two most overrepresented sequences in ERG-, ETV1-, or ETV4-bound regions were identical (AGGAA and TGA[C/G]TCA). AGGAA is a weak ETSbinding site similar to those previously identified in specific ETS-bound enhancers that are supported by cooperative interactions with neighboring transcription factors. The sequence TGA/C/G)TCA is not an ETS-binding site and may instead represent a binding site for a factor that collaborates with ERG, ETV1, and ETV4 for a specific oncogenic function. The nononcogenic ETS proteins GABPA and ETS1 were not found to be associated with either of these sequences. In GABPA-bound regions, the most enriched sequence was CCGGAAGT, identical to the sequence found in GABPA-bound regions in T cells and indicative of housekeeping promoter targets (Hollenhorst et al. 2007). ETS1-bound regions had the highest enrichment for the sequence TGGGANNTGTAGT, a sequence previously identified in ETS1-specific promoters in T cells (Hollenhorst et al. 2007). Thus, a distinct set of sequence motifs is common to regions bound by oncogenic ETS proteins, but not other ETS family members.
To further define DNA sequence motifs common to ERG, ETV1, and ETV4 genomic occupancy, MEME was used to identify sequences overrepresented in regions occupied by all three proteins (Fig. 3B). The sequences TGA/C/G)TCA and AGGAA were identified again, along with a third sequence, AGGAAGTGAC. Furthermore, direct searches confirmed that each sequence occurred in bound regions more often than expected by chance. The third sequence represents a composite of the second motif juxtaposed to the first four nucleotides of the first motif. A frequency distribution of the spacing and orientation of AGGAA and TGANTCA in regions bound by ERG indicates that this particular spacing and orientation is indeed the most common (Fig. 3C). Thus, the juxtaposition of a weak ETS-binding site and the sequence TGA(C/G)TCA is a hallmark of oncogenic ETS protein binding.

\section{Co-occupancy of AP-1 with an oncogenic ETS protein}

The TGA/C/G)TCA sequence found in regions occupied by oncogenic ETS proteins matches exactly the consensus binding sequence for the AP-1 class of transcription factors. JUN homodimers or JUN/FOS heterodimers constitute AP-1-binding activity (Chinenov and Kerppola 2001). To test whether AP-1 co-occupies these regions with oncogenic ETS proteins, various AP-1 subunit antibodies were screened in PC3 cell ChIP for the ability to enrich an ETV4-bound region (Supplemental Fig. S2A). ChIP with a JUND antibody enriched this region. ChIP-seq in PC3 cells using this antibody identified 2973 bound regions. These bound regions overlapped with $31 \%$ of the ETV4bound regions, a 145-fold enrichment over the random expectation (Fig. 4A). The most frequent spacing and orientation of ETS and AP-1 sequences in regions co-occupied by ETV4 and JUND was the same as in ERG-bound regions (Supplemental Fig. S2B). JUND-bound regions in PC3 cells also overlapped with ERG- and ETV1-bound regions in RWPE-1 cells more often than regions bound by ETS1 and GABPA (Fig. 4A), indicating that AP-1 occupancy correlates with specific binding of oncogenic ETS proteins.

\section{PLAU is a target of oncogenic ETS proteins}

Composite ETS/AP-1-binding sequences have been previously identified as promoter and enhancer regulatory elements (Chinenov and Kerppola 2001). The human PLAU gene, encoding the extracellular matrix remodeler urokinase plasminogen activator ( $\mathrm{uPA})$, is regulated by an enhancer mapped by reporter assays to a position $2 \mathrm{~kb}$ upstream of the TSS (Nerlov et al. 1991). Proper regulation of this enhancer requires a composite ETS/AP-1-binding sequence (AGGAAATGA) with the same spacing and orientation as the sequence identified in Figure 3B (Nerlov et al. 1992). Mice have two ETS/AP-1-regulated PLAU enhancers at positions -2 and $-7 \mathrm{~kb}$ (D'Orazio et al. 1997). We identified two regions bound by ETV4 and JUND near the human PLAU TSS (Fig. 4B). One region was at the same position as the previously mapped human enhancer, and the other was $2.5 \mathrm{~kb}$ further upstream and may be the human equivalent of the second mouse enhancer. Flag antibody ChIP-seq of Flag-ERG and Flag-ETV1 in RWPE-1 


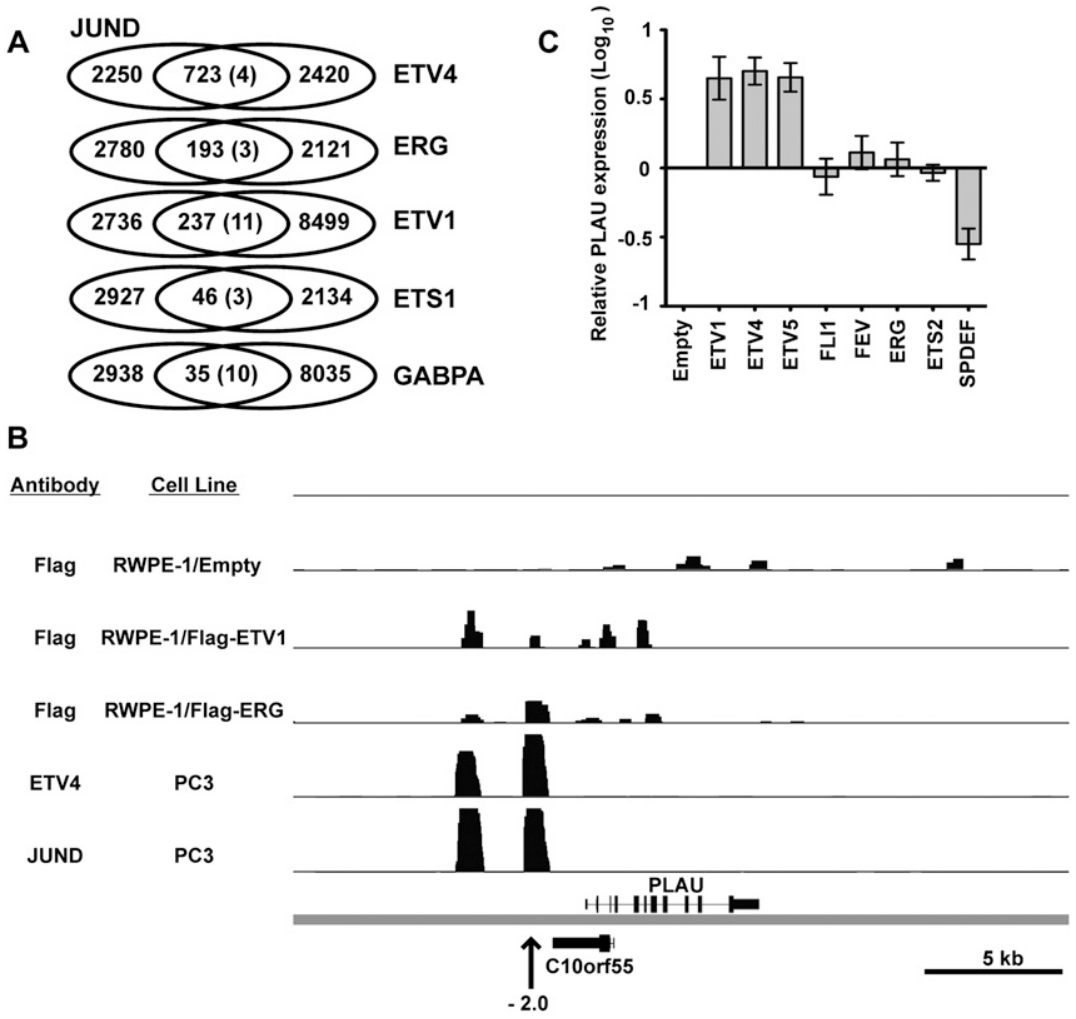

Figure 4. AP-1 and oncogenic ETS proteins cooccupy genomic regions, including the PLAU enhancers. (A) JUND-bound regions determined by ChIP-seq of endogenous proteins in PC3 cells are overlapped with ETS-bound regions in either PC3 cells (ETV4) or RWPE-1 cells (ERG, ETV1, ETS1, and GABPA). The random prediction is shown in parentheses as in Figure 2C. $(B)$ ChIP-seq binding peaks are displayed in a $30-\mathrm{kb}$ region of the human genome surrounding the PLAU gene (hg18, chr10:75,329,500$75,359,500)$ using the Integrated Genome Browser (http://igb.bioviz.org). PLAU is transcribed from left to right, and C10orf55 is transcribed from right to left. The $Y$-axis graphs log-transformed $P$-values based on ChIP-seq with the indicated antibody and cell line. Each track is shown in the same scale (0-150). An arrow marks the previously mapped PLAU enhancer at $-2 \mathrm{~kb}$. $(C)$ PEA3 subfamily members, but not other ETS proteins, activate PLAU in RWPE-1 cells in normal growth medium. $P L A U$ gene expression in RWPE-1 cells overexpressing the indicated ETS proteins was measured by qRT-PCR, normalized to the mean of control transcript 18s rRNA, and reported on a log scale relative to levels in cells expressing empty vector control (shown in lane 1). Results are the mean and SEM of three independent replicates. cells indicated binding of these same two enhancer regions. Thus, two PLAU enhancers represent direct targets for oncogenic ETS proteins.

Regulation of the endogenous PLAU locus by oncogenic ETS proteins was tested in RWPE-1 cells expressing various ETS proteins (Fig. 4C). Each of the oncogenic PEA3 subfamily members (ETV1, ETV4, and ETV5) elevated $P L A U$ mRNA levels as detected by quantitative RT-PCR (qRT-PCR). In contrast, the ETS protein SPDEF repressed $P L A U$, corresponding to its tumor-suppressive role in prostate cells (Gu et al. 2007; Turner et al. 2011). However, ERG had no affect on PLAU expression, similar to the nononcogenic ETS proteins FEV, FLI1, and ETS2.

\section{Activation of RAS-MAPK target genes in the absence of pathway activation}

ETS/AP-1-binding sites, including those in the PLAU enhancers, can act as response elements for the RAS/MAPK signaling pathway in cell-based assays (Nerlov et al. 1992; Stacey et al. 1995). We next tested the role of RAS/MAPK signaling in the regulation of $P L A U$ by ETS proteins. Normal growth medium for RWPE-1 cells was supplemented by recombinant epidermal growth factor (EGF) and bovine pituitary extract. RWPE-1 cells had high ERK phosphorylation in this medium, indicating an active RAS/ MAPK pathway (Fig. 5A). The addition of the MEK inhibitor U0126 or the withdrawal of supplements resulted in a loss of ERK phosphorylation. PLAU expression levels corresponded to the activity of this pathway, as levels decreased with U0126 addition or supplement withdrawal and increased with the addition of the RAS/MAPK pathway agonist PMA (Fig. 5B). Thus, similar to other cell types, PLAU expression in prostate cells is regulated by the RAS/MAPK pathway, likely via one or more of the ETS transcription factors that are expressed in normal prostate and activated by RAS/MAPK signaling (ELK1, ELK3, ELK4, ETS1, ETS2, and GABPA).

The activation of the RAS/MAPK pathway by the supplements in RWPE-1 growth medium may explain why ERG had no effect on PLAU expression (Fig. 4C). To test the role of ETS proteins in the absence of MEK/ERK signaling, PLAU expression was monitored in RWPE-1 cells in the presence of U0126 or in medium lacking supplements. In both cases, expression of the oncogenic ETS proteins ERG and ETV1 increased PLAU levels (Fig. 5C). Intriguingly, the prostate tumor suppressor SPDEF could no longer repress PLAU expression in the absence of MAPK signaling, indicating that the function of SPDEF may be to replace a RAS-responsive ETS protein and attenuate transcriptional activation.

To test the ability of ERG and ETV1 to activate other RAS/MAPK target genes in the absence of pathway activation, global gene expression changes were monitored by microarray. In control RWPE-1 cells (empty vector), treatment with the MEK inhibitor U0126 decreased the expression of 769 genes and increased the expression of 608 genes (based on a mean change $>1$.7-fold and $P$-value $<0.001$ ). A comparison with a set of genes repressed by MEK inhibition in other cell types (Pratilas et al. 2009) found 43 of 44 genes also down-regulated by U0126 in RWPE-1 (Supplemental Table S2). The introduction of exogenous 
ERG or ETV1 to RWPE-1 cells in the continued presence of U0126 resulted in a striking reversal of the U0126-mediated gene expression changes. ERG overexpression restored the RAS/MAPK gene expression program, as evidenced by up-regulation of $42 \%$ (320) of the U0126-repressed genes and down-regulation of $43 \%$ (259) of the U0126-activated genes (Fig. 5D; Supplemental Table S3). In contrast, only $5 \%(38)$ of the repressed genes were further down-regulated and $4 \%(22)$ of the activated genes were further activated. ETV1 overexpression had a similar ability to reverse effects of MEK inhibition, as shown by up-regulation of $42 \%$ of U0126-repressed genes (8\% of activated genes) and down-regulation of $52 \%$ of U0126-activated genes $16 \%$ of repressed genes). This mode of regulation was confirmed for a subset of genes by qRT-PCR (Supplemental Fig. S3). Thus, introduction of ERG or ETV1 expression into normal prostate cells activates a RAS/MAPK gene expression program in the absence of ERK activation.

To test for direct regulation by oncogenic ETS proteins, the RAS/MAPK-regulated gene list was compared with a list of genes identified as ETS targets by genomic occupancy. These putative direct targets of ERG and ETV1 were 3.1-fold enriched for genes down-regulated by MEK inhibition and 1.5-fold enriched for genes up-regulated by
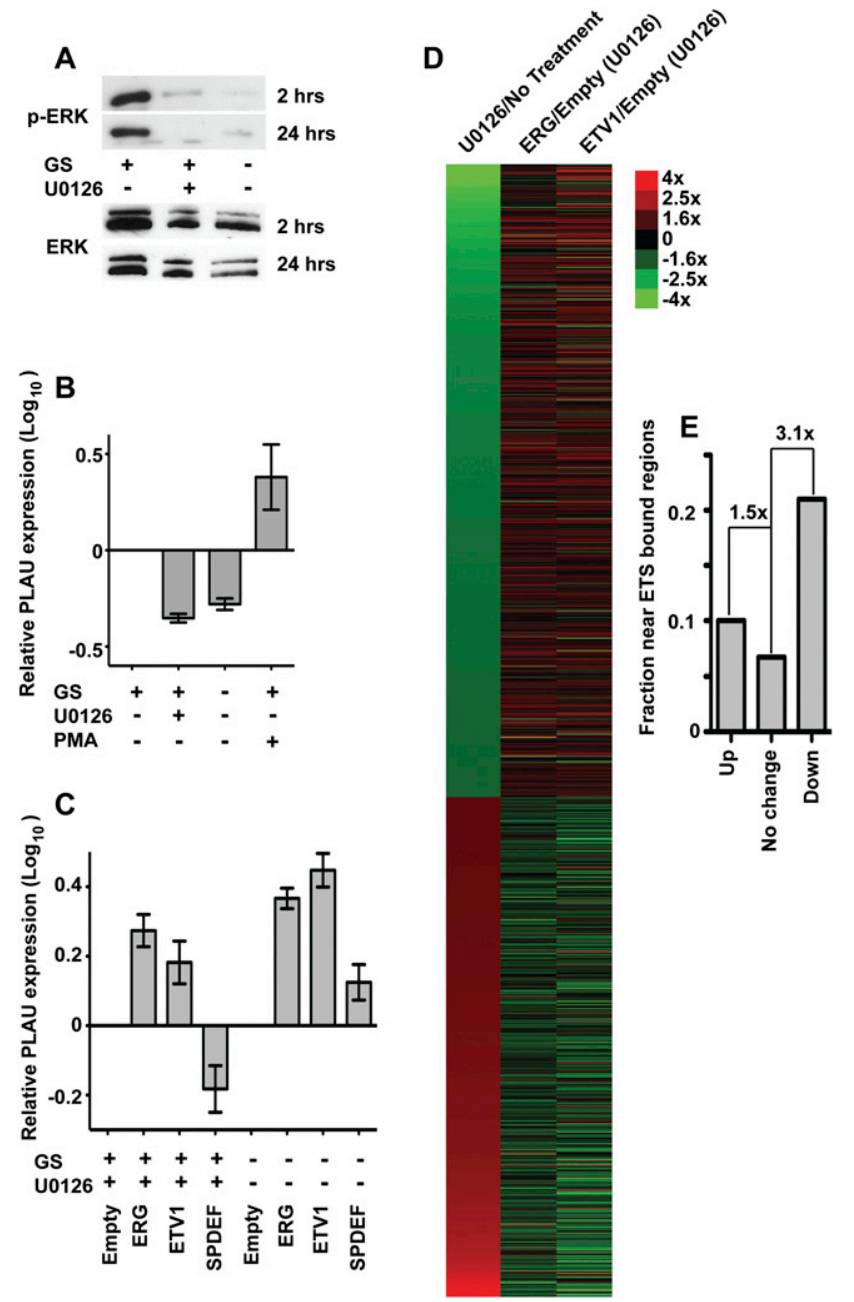

MEK inhibition, compared with all other genes (Fig. 5E). This indicates that occupancy of oncogenic ETS proteins is predictive of RAS/MAPK pathway target genes, particularly those activated by the pathway.

\section{Discussion}

We report that ETS genes associated with chromosomal rearrangements in prostate cancer represent a functionally distinct subset of the ETS family that links to prostate cell migration behavior. Furthermore, these oncogenic ETS factors occupied a common set of genomic targets that differ from targets of other ETS proteins and are defined by closely juxtaposed ETS- and AP-1-binding motifs. Gene expression analysis indicates that de novo expression of oncogenic ETS proteins can substitute for RAS/MAPK signaling. This pattern was observed specifically with PLAU, whose gene product, uPA, relates to cell migration by its effect on extracellular matrix remodeling.

\section{Specificity of ETS proteins in prostate cancer}

Our understanding of the specific biological functions of ETS genes is challenged by the overlapping DNA sequence preference and extensive coexpression of ETS transcription factors. This specificity problem is recreated in ETS-

Figure 5. ERG and ETV1 activated a RAS/MAPK gene expression program in the absence of ERK activation. (A) Immunoblots identified protein levels in RWPE-1 whole-cell extracts using either an antibody to Y-204 phosphorylated ERK (p-ERK) or an anti-ERK antibody as indicated. Cells were cultured in the presence or absence of $10 \mu \mathrm{M}$ U0126 or growth supplements (GS; EGF and bovine pituitary extract) for the time indicated. (B) $P L A U$ gene expression was measured as described in Figure 4C from RWPE- 1 cells grown in the presence or absence of the indicated treatments for $6 \mathrm{~h}$. Results are reported relative to expression in normal growth medium (shown in lane 1) and are the mean and SEM of two independent replicates. (C) PLAU expression measured as in $B$ from RWPE-1 cells overexpressing the indicated ETS protein and treated as indicated. Data are reported relative to expression in cells with an empty vector, not overexpressing an ETS protein (Empty). Results are the mean and SEM of four independent replicates. $(D)$ A heat map shows mean gene expression changes in four replicates each of three microarray experiments. Genes displayed are those with a $>1.7$-fold change and $P$-value $<0.001$ in empty vector RWPE-1 cells treated with U0126 compared with untreated, empty vector RWPE-1 cells. Genes are rank-ordered from most downregulated by U0126 to most up-regulated by U0126 (shown in lane 1). Gene expression changes in cells overexpressing ERG or ETV1 in the presence of U0126 compared with empty vector (Empty) cells in the presence of U0126 are shown in lanes 2 and 3 , respectively. Red indicates up-regulated genes and green indicates down-regulated genes. $(E)$ The fraction of genes in the indicated categories that have neighboring ERG- and ETV1bound regions. Up and down categories include genes that increase or decrease expression, respectively, when U0126 is added to empty vector RWPE-1 cells as shown in lane 1 in $D$. Unchanged refers to all remaining genes. A neighboring gene is that with the closest TSS to a bound region. Fold enrichment over unchanged genes is shown above. 
driven disease states. Chromosomal rearrangements in prostate cancer result in overexpression of either full-length or N-terminally truncated versions of one of only four ETS transcription factors (Kumar-Sinha et al. 2008). Overexpression of each of these four ETS genes has been reported to increase invasion or migration of cell lines derived from normal prostate (Cai et al. 2007; Tomlins et al. 2007, 2008; Helgeson et al. 2008; Hollenhorst et al. 2011b), In this report, we confirmed this effect and demonstrated that other ETS family members, including FLI1 and FEV, which are most similar to $E R G$, failed to enhance migration. Furthermore, this subset of oncogenic ETS proteins differed from nononcogenic ETS proteins in both genomic occupancy and the regulation of RAS/MAPK target genes in the absence of RAS/MAPK signaling. Thus, the ETS oncogenes ERG, ETV1, ETV4, and ETV5 comprise a functionally distinct subset of the ETS family. However, the similarity of these ETS oncogenes was context-dependent, as the PEA3 subfamily members, but not ERG, could also respond to RAS/MAPK signaling and further activate RASresponsive genes (Fig. $4 \mathrm{C}$ ).

Based on these findings, we propose a competition model (Fig. 6) in which a change in the ETS protein bound at ETS/AP-1 target sites can alter both the expression level and RAS responsiveness of target genes. In cells, a coupled equilibrium would coordinate multiple ETS factors and genomic ETS-binding sites. Fluctuations in ETS protein levels would vary the relative occupancy time of each ETS family member. Thus, an exogenous, oncogenic ETS protein would compete for genomic binding sites with endogenous ETS proteins. In this model, the difference in transcriptional activity between the original and replacement ETS protein results in a change in gene expression that contributes to tumor progression. This model is consistent with the ability to subcategorize prostate tumors by the expression levels of both oncogenic and nononcogenic ETS genes (Kunderfranco et al. 2010).

A functional partnership with AP-1 would bias the competition for ETS/AP-1 RAS-responsive elements. Thus, the ability of an ETS factor to bind sites in vivo would depend on both cooperative DNA-binding affinity and
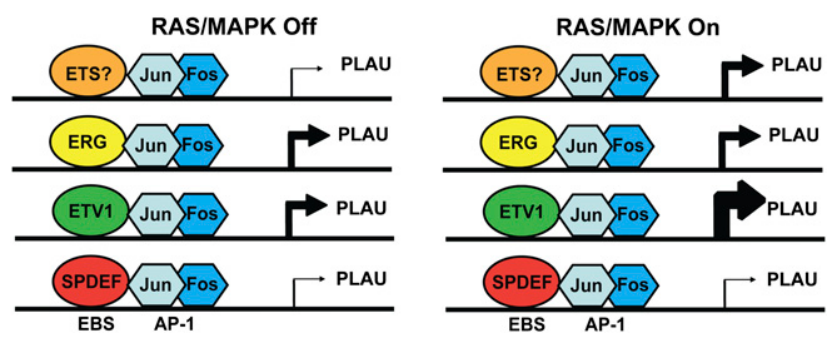

Figure 6. A model uses $P L A U$ to represent the regulation of ETS/AP-1-regulated, RAS/MAPK target genes by ETS transcription factors. An unidentified endogenous ETS protein (ETS?) binds ETS/AP-1 sequence elements, but only activates gene expression when the RAS/MAPK pathway is active. The oncogenic ETS proteins ERG and ETV1 can activate expression when the RAS/MAPK pathway is off. ETV1 can superactivate when the RAS/MAPK pathway is on. SPDEF attenuates RAS/MAPKmediated transcriptional activation. relative protein concentration. Multiple ETS proteins can activate transcription via ETS/AP-1 sequences in response to RAS signaling, with the best-studied examples including members of the ETS (ETS1 and ETS2) and PEA3 (ETV1, ETV4, and ETV5) subfamilies (Yordy and Muise-Helmericks 2000). However, prior techniques failed to conclusively identify which ETS family members function in collaboration with AP-1 in any particular cell type. Here, we present an identification of a genome-wide association between ETS proteins and AP-1. These data suggest that AP-1 plays a role in the selective recruitment of oncogenic ETS proteins to the genome. In vitro DNA-binding studies support this model, as the ETS proteins ETV1, ERG, and FLI1, but not ETS2 and SPI1, are reported to bind DNA cooperatively with AP-1 (Verger et al. 2001; Kim et al. 2006). SPDEF and EHF are candidates for occupancy in normal prostate because they are the two most highly expressed ETS family members in this tissue (Hollenhorst et al. 2004) and knockdown of these factors increases survival and migration of prostate cancer cell lines (Gu et al. 2007; Cangemi et al. 2008; Turner et al. 2011). However, our ability to overexpress SPDEF and further decrease PLAU expression (Fig. 4C) indicates that we can drive increased occupancy in RWPE-1 cells.

Oncogenic ETS proteins might bind multiple target classes that are regulated differently. ERG and ETV1 targets overlapped significantly more often than nononcogenic ETS targets; however, more than one-half of targets did not overlap, indicating that ERG and ETV1 may also have unique, unidentified functions. The direct binding of ERG or ETV1 is primarily associated with the activation, rather than repression, of RAS/MAPK-regulated genes (Fig. 5E). One example is $P L A U$, a gene previously identified as a direct target of ERG in prostate cells (Tomlins et al. 2008; $\mathrm{Yu}$ et al. 2010). However, at all targets, ETV4 occupancy correlated equally with both activation and repression (Fig. 2D). Therefore, an additional target class could be genes regulated by AR. Previous studies using ChIP-seq to identify ERG targets in both prostate cell lines and tumors revealed a $44 \%$ overlap between ERG and AR occupancy (Wei et al. 2010; Yu et al. 2010). This ERG occupancy is associated with attenuation of AR transcriptional activation (Yu et al. 2010).

\section{Role of ETS genes in other cancers}

Remarkably, these four ETS genes associated with prostate cancer only partially overlap with the group of ETS genes implicated in Ewing's sarcoma (Fig. 1A). In Ewing's sarcoma, fusion oncogenes, generated by chromosome translocation, encode the $\mathrm{N}$ terminus of the EWS protein and the DNA-binding domains of ERG, FLI1, FEV, ETV1, or ETV4 (Mackintosh et al. 2010). The difference between the prostate- and Ewing's sarcoma-related subsets of ETS proteins is likely due to the inclusion of the $\mathrm{N}$ terminus of EWS, which imparts novel transcriptional functions and is required for transformation (May et al. 1993). Interestingly, a cooperative DNA-binding interaction between EWS-FLI1 and AP-1 is important for transformation (Kim et al. 2006), suggesting that the ETS fusions associated with Ewing's 
sarcoma and prostate cancer-associated ETS proteins may both function by targeting ETS/AP1 sequences.

The ETS/AP-1-regulated, RAS-responsive, gene expression program, which we defined in prostate cancer, may be important more broadly. ETV1 is expressed at high levels in GIST and melanoma, cancers that frequently have mutations (KIT and BRAF, respectively) that activate the RAS/MAPK pathway (Chi et al. 2010; Jane-Valbuena et al. 2010). Furthermore, ChIP-seq of ETV1 in GIST (Chi et al. 2010) identified an ETS-binding sequence (CAGGAAG) similar to the most overrepresented sequence identified in ETV1-bound regions in this study (Fig. 3A). Interestingly, melanoma and colon cancer cell lines with activating mutations in BRAF (V600E) express a set of MEK-regulated targets similar to those presented here (Supplemental Table S2; Pratilas et al. 2009). Among these targets are ETV1, ETV4, and ETV5. Our data indicate that these PEA3 subfamily members provide stronger transcriptional activation than other ETS proteins when the RAS/MAPK pathway is active (Fig. 4C). This suggests a positive feedback loop for PEA3 ETS genes in these cancers, similar to that recently described for ERG in prostate cancer (Mani et al. 2011). PEA3 subfamily expression is also increased in cancer cells by protein stabilization. In GIST, ETV1 protein levels are stabilized by MEK activity (Chi et al. 2010). In addition, PEA3 subfamily members are targeted by COP1 for ubiquitin-dependent degradation via a domain that is usually lost in prostate cancer chromosome rearrangements (Vitari et al. 2011). Our model suggests that cancers with mutations that activate the RAS/ MAPK signaling pathway could superactivate this pathway by increased expression or stabilization of PEA3 subfamily members. However, ERG overexpression would not increase the expression of RAS/MAPK targets when the pathway is activated (Fig. 6). These results are supported by the failure to discover ERG overexpression in any cancer that is commonly associated with activating mutations in the RAS/MAPK pathway. Furthermore, superactivation of a RAS/MAPK-regulated gene expression program may relate to the stronger invasive growth of melanoma compared with early stage prostate cancer.

Taken together, our findings suggest that oncogenic ETS transcription factors can replace RAS/MAPK pathway function by activating target genes regulated by ETS/AP-1 sequences. This signaling mimicry is consistent with the relatively infrequent occurrence of RAS and RAF mutations in early androgen-sensitive prostate cancers. As cancers progress to androgen independence, many express the androgen-responsive TMPRSS2-ERG fusion at reduced levels (Hermans et al. 2006; Bonaccorsi et al. 2009). Interestingly, the transition to androgen independence has also been linked to the activation of the RAS/MAPK signaling pathway (Weber and Gioeli 2004; Taylor et al. 2010), thus providing for ongoing activation of the RAS/MAPK gene expression program. By providing a bypass of signaling activation events, ETS-driven cancers may require new therapeutic angles that can be directed at the transcriptional regulatory machinery.

\section{Materials and methods}

\section{Cell culture, retroviral expression, and migration assays}

PC3 and RWPE-1 cells were obtained from American Type Culture Collection and cultured accordingly. The ETV4-expressing retrovirus was described previously (Hollenhorst et al. 2011b). Remaining retroviral expression plasmids were made by the same method using primers provided as Supplemental Material. Empty vector was pQCXIH (Clontech). Retroviral expression plasmids were cotransfected with vesicular stomatitis virus-G glycoprotein and gag/pol packaging plasmids into 293 EBNA cells to create retroviruses. Retroviruses were added to RWPE-1 cells with $8 \mathrm{mg} / \mathrm{mL}$ polybrene for $2 \mathrm{~h}$, before replacement with growth medium. After $24 \mathrm{~h}$, cells were maintained under hygromycin selection (250 $\mu \mathrm{g} / \mathrm{mL})$.

Migration assays were performed as previously described (Hollenhorst et al. 2011b). In short, $5 \times 10^{4}$ cells were plated into a Boyden chamber (BD Biosciences) in the absence of growth supplements and allowed to migrate through $8-\mu \mathrm{m}$ pores to normal growth medium for $60 \mathrm{~h}$. Cells on the outer side of the chamber were stained and counted.

\section{Protein immunoblots and ChIP}

Whole-cell extracts of equivalent cell number were run on $10 \%$ SDS-PAGE gels and blotted to nitrocellulose. Proteins were detected with either anti-Flag M2 (Sigma Life Science), p-ERK (sc-7383, Santa Cruz Biotechnologies), or ERK1 (sc-94, Santa Cruz Biotechnologies) antibodies.

ChIP was performed as described previously (Hollenhorst et al. 2007) with the exception that Flag ChIP beads were washed with a more stringent wash buffer containing $500 \mathrm{mM} \mathrm{LiCl}$. Antibodies used for ChIP included anti-Flag M2 (Sigma Life Sciences), ETV4 (ARP32262, Aviva Systems Biology), or Santa Cruz Biotechnologies antibodies ETS1 (sc-350), GABPA (sc-22810), or JUND (sc74). Primer sequences are available in the Supplemental Material.

\section{ChIP-seq and analysis}

ChIP-seq was performed as described previously (Hollenhorst et al. 2009). In short, each ChIP sample was pooled from at least three independent ChIP experiments. Libraries were prepared using Illumina's ChIP-seq kit. Thirty-six-base-pair single-end reads were generated using a Genome Analyzer II and standard pipeline software (Illumina). Useq software (Nix et al. 2008) was used to analyze ChIP-seq data as described previously (Hollenhorst et al. 2009). Reads from opposite strands were adjusted by the peak shift. Peak shifts were JunD, 250 bp; ETV1, 100 bp; ETS1, 270 bp; ERG, $270 \mathrm{bp}$; ETV4, $260 \mathrm{bp}$; and GABPA, $250 \mathrm{bp}$. Sliding windows were selected to be approximately twice the peak shift (500 bp for all analyses except ETV1, which was 250 bp). Significance was determined by calculating a binomial $P$-value for each window and controlled for multiple testing by calculating an empirical FDR. Bound regions were overlapped with the IntersectRegions tool with no gap. The ChIP-seq raw data sets and processed peak files are available for download from NCBI's Gene Expression Omnibus (GEO; http://www.ncbi.nlm.nih.gov/geo), accession number GSE29808.

Default settings were used for MEME (http://meme.sdsc.edu) except the maximum motif length was set to 13. Results in Figure $3 \mathrm{~A}$ used the 250 regions from each bound region list with the highest log-transformed binomial $P$-value. Analysis using all bound regions returned a similar result. GoMiner was used with default settings except "Evidence level 4" and "All/gene ontology" were selected. All RefSeq genes were used as the total gene list. 


\section{$q R T-P C R$ and microarray}

Reverse transcription used gene-specific primers and SuperScript III reverse transcriptase (Invitrogen). qPCR was performed using SYBR FAST mix (Kapa Biosystems) on an Eppendorf RealPlex2 MasterCycler using serially diluted PCR products as standard curves. Transcript levels were normalized to GAPDH levels. A second normalization standard, EEF1A, provided essentially the same results (data not shown). Normalized expression levels are presented as a $\log _{10}$-transformed ratio to the control (empty vector). Primer sequences are available in the Supplemental Material.

Microarray data sets are available for download from GEO using accession number GSE29438. Four independent samples of total RNA were isolated by RNeasy kit (Qiagen) for each microarray experiment. Total RNA was primed with oligo-dT and converted to cDNA using a Double-Stranded cDNA Synthesis kit (Invitrogen). cDNA was labeled using the Dual-Color Labeling kit (Roche Nimblegen) and hybridized to a Nimblegen Homo sapiens HG18 expression array (12x135k) using a Hybridization Systems kit (Roche Nimblegen). Image acquisition used an Axon GenePix 4200A scanner at 5- $\mu \mathrm{m}$ resolution. Raw signal intensities were extracted with Nimblescan 2.6 software (Roche Nimblegen) and were quantile-normalized.

\section{Acknowledgments}

We thank University of Utah colleagues Brian Dalley for assistance with Illumina sequencing and David Nix for advice regarding ChIP-seq analysis. Thanks to the Indiana University Center for Genomics and Bioinformatics for microarray hybridization and data analysis. This work was supported by the National Institutes of Health (GM38663 to B.J.G., and CA42014 to the Huntsman Cancer Institute for support of core facilities). B.J.G. acknowledges funding from the Huntsman Cancer Institute/ Huntsman Cancer Foundation and the Prostate Cancer Foundation. P.C.H. acknowledges support from the Indiana University School of Medicine and the Walther Cancer Foundation.

\section{References}

Baert JL, Beaudoin C, Monte D, Degerny C, Mauen S, de Launoit Y. 2007. The $26 \mathrm{~S}$ proteasome system degrades the ERM transcription factor and regulates its transcription-enhancing activity. Oncogene 26: 415-424.

Bailey TL, Elkan C. 1994. Fitting a mixture model by expectation maximization to discover motifs in biopolymers. Proc Int Conf Intell Syst Mol Biol 2: 28-36.

Bello D, Webber MM, Kleinman HK, Wartinger DD, Rhim JS. 1997. Androgen responsive adult human prostatic epithelial cell lines immortalized by human papillomavirus 18. Carcinogenesis 18: 1215-1223.

Bonaccorsi L, Nesi G, Nuti F, Paglierani M, Krausz C, Masieri L, Serni S, Proietti-Pannunzi L, Fang Y, Jhanwar SC, et al. 2009. Persistence of expression of the TMPRSS2:ERG fusion gene after pre-surgery androgen ablation may be associated with early prostate specific antigen relapse of prostate cancer: preliminary results. J Endocrinol Invest 32: 590-596.

Bos JL. 1989. ras oncogenes in human cancer: a review. Cancer Res 49: 4682-4689.

Cai C, Hsieh CL, Omwancha J, Zheng Z, Chen SY, Baert JL, Shemshedini L. 2007. ETV1 is a novel androgen receptorregulated gene that mediates prostate cancer cell invasion. Mol Endocrinol 21: 1835-1846.

Cangemi R, Mensah A, Albertini V, Jain A, Mello-Grand M, Chiorino G, Catapano CV, Carbone GM. 2008. Reduced expression and tumor suppressor function of the ETS tran- scription factor ESE-3 in prostate cancer. Oncogene 27: 2877-2885.

Carver BS, Tran J, Gopalan A, Chen Z, Shaikh S, Carracedo A, Alimonti A, Nardella C, Varmeh S, Scardino PT, et al. 2009. Aberrant ERG expression cooperates with loss of PTEN to promote cancer progression in the prostate. Nat Genet 41: 619-624.

Charlot C, Dubois-Pot H, Serchov T, Tourrette Y, Wasylyk B. 2010. A review of post-translational modifications and subcellular localization of Ets transcription factors: possible connection with cancer and involvement in the hypoxic response. Methods Mol Biol 647: 3-30.

Chi P, Chen Y, Zhang L, Guo X, Wongvipat J, Shamu T, Fletcher JA, Dewell S, Maki RG, Zheng D, et al. 2010. ETV1 is a lineage survival factor that cooperates with KIT in gastrointestinal stromal tumours. Nature 467: 849-853.

Chinenov Y, Kerppola TK. 2001. Close encounters of many kinds: Fos-Jun interactions that mediate transcription regulatory specificity. Oncogene 20: 2438-2452.

Davies H, Bignell GR, Cox C, Stephens P, Edkins S, Clegg S, Teague J, Woffendin H, Garnett MJ, Bottomley W, et al. 2002. Mutations of the BRAF gene in human cancer. Nature 417: 949-954.

D'Orazio D, Besser D, Marksitzer R, Kunz C, Hume DA, Kiefer B, Nagamine Y. 1997. Cooperation of two PEA3/AP1 sites in uPA gene induction by TPA and FGF-2. Gene 201: 179-187.

Ellett F, Kile BT, Lieschke GJ. 2009. The role of the ETS factor erg in zebrafish vasculogenesis. Mech Dev 126: 220-229.

Gu X, Zerbini LF, Otu HH, Bhasin M, Yang Q, Joseph MG, Grall F, Onatunde T, Correa RG, Libermann TA. 2007. Reduced PDEF expression increases invasion and expression of mesenchymal genes in prostate cancer cells. Cancer Res 67: 4219-4226.

Helgeson BE, Tomlins SA, Shah N, Laxman B, Cao Q, Prensner JR, Cao X, Singla N, Montie JE, Varambally S, et al. 2008. Characterization of TMPRSS2:ETV5 and SLC45A3:ETV5 gene fusions in prostate cancer. Cancer Res 68: 73-80.

Hermans KG, van Marion R, van Dekken $H$, Jenster G, van Weerden WM, Trapman J. 2006. TMPRSS2:ERG fusion by translocation or interstitial deletion is highly relevant in androgen-dependent prostate cancer, but is bypassed in latestage androgen receptor-negative prostate cancer. Cancer Res 66: 10658-10663.

Hollenhorst PC, Jones DA, Graves BJ. 2004. Expression profiles frame the promoter specificity dilemma of the ETS family of transcription factors. Nucleic Acids Res 32: 5693-5702.

Hollenhorst PC, Shah AA, Hopkins C, Graves BJ. 2007. Genomewide analyses reveal properties of redundant and specific promoter occupancy within the ETS gene family. Genes Dev 21: 1882-1894.

Hollenhorst PC, Chandler KJ, Poulsen RL, Johnson WE, Speck NA, Graves BJ. 2009. DNA specificity determinants associate with distinct transcription factor functions. PLoS Genet 5: e1000778. doi: 10.1371.journal.pgen.1000778.

Hollenhorst PC, McIntosh LP, Graves BJ. 2011a. Genomic and biochemical insights into the specificity of ETS transcription factors. Annu Rev Biochem 80: 437-471.

Hollenhorst PC, Paul L, Ferris MW, Graves BJ. 2011b. The ETS gene ETV4 is required for anchorage-independent growth and a cell proliferation gene expression program in PC3 prostate cells. Genes Cancer 1: 1044-1052.

Jane-Valbuena J, Widlund HR, Perner S, Johnson LA, Dibner AC, Lin WM, Baker AC, Nazarian RM, Vijayendran KG, Sellers WR, et al. 2010. An oncogenic role for ETV1 in melanoma. Cancer Res 70: 2075-2084.

Kim S, Denny CT, Wisdom R. 2006. Cooperative DNA binding with AP-1 proteins is required for transformation by EWSEts fusion proteins. Mol Cell Biol 26: 2467-2478. 
King JC, Xu J, Wongvipat J, Hieronymus $\mathrm{H}$, Carver BS, Leung DH, Taylor BS, Sander C, Cardiff RD, Couto SS, et al. 2009. Cooperativity of TMPRSS2-ERG with PI3-kinase pathway activation in prostate oncogenesis. Nat Genet 41: 524-526.

Kumar-Sinha C, Tomlins SA, Chinnaiyan AM. 2008. Recurrent gene fusions in prostate cancer. Nat Rev Cancer 8: 497-511.

Kunderfranco P, Mello-Grand M, Cangemi R, Pellini S, Mensah A, Albertini V, Malek A, Chiorino G, Catapano CV, Carbone GM. 2010. ETS transcription factors control transcription of $\mathrm{EZH} 2$ and epigenetic silencing of the tumor suppressor gene Nkx3.1 in prostate cancer. PLOS ONE 5: e10547. doi: 10.1371/journal.pone.0010547.

Lin C, Yang L, Tanasa B, Hutt K, Ju BG, Ohgi K, Zhang J, Rose DW, Fu XD, Glass CK, et al. 2009. Nuclear receptor-induced chromosomal proximity and DNA breaks underlie specific translocations in cancer. Cell 139: 1069-1083.

Mackintosh C, Madoz-Gurpide J, Ordonez JL, Osuna D, HerreroMartin D. 2010. The molecular pathogenesis of Ewing's sarcoma. Cancer Biol Ther 9: 655-667.

Mani RS, Tomlins SA, Callahan K, Ghosh A, Nyati MK, Varambally S, Palanisamy N, Chinnaiyan AM. 2009. Induced chromosomal proximity and gene fusions in prostate cancer. Science 326: 1230.

Mani RS, Iyer MK, Cao Q, Brenner JC, Wang L, Ghosh A, Cao X, Lonigro RJ, Tomlins SA, Varambally S, et al. 2011. TMPRSS2ERG-mediated feed-forward regulation of wild-type ERG in human prostate cancers. Cancer Res 71: 5387-5392.

May WA, Lessnick SL, Braun BS, Klemsz M, Lewis BC, Lunsford LB, Hromas R, Denny CT. 1993. The Ewing's sarcoma EWS/ FLI-1 fusion gene encodes a more potent transcriptional activator and is a more powerful transforming gene than FLI-1. Mol Cell Biol 13: 7393-7398.

McLaughlin F, Ludbrook VI, Cox J, von Carlowitz I, Brown S, Randi AM. 2001. Combined genomic and antisense analysis reveals that the transcription factor Erg is implicated in endothelial cell differentiation. Blood 98: 3332-3339.

Nerlov C, Rorth P, Blasi F, Johnsen M. 1991. Essential AP-1 and PEA3 binding elements in the human urokinase enhancer display cell type-specific activity. Oncogene 6: 1583-1592.

Nerlov C, De Cesare D, Pergola F, Caracciolo A, Blasi F, Johnsen M, Verde P. 1992. A regulatory element that mediates cooperation between a PEA3-AP-1 element and an AP-1 site is required for phorbol ester induction of urokinase enhancer activity in HepG2 hepatoma cells. EMBO J 11: 4573-4582.

Nix DA, Courdy SJ, Boucher KM. 2008. Empirical methods for controlling false positives and estimating confidence in ChIP-seq peaks. BMC Bioinformatics 9: 523. doi: 10.1186/ 1471-2105-9-523.

Pratilas CA, Taylor BS, Ye Q, Viale A, Sander C, Solit DB, Rosen N. 2009. (V600E)BRAF is associated with disabled feedback inhibition of RAF-MEK signaling and elevated transcriptional output of the pathway. Proc Natl Acad Sci 106: 4519-4524.

Shen MM, Abate-Shen C. 2010. Molecular genetics of prostate cancer: new prospects for old challenges. Genes Dev 24: 1967-2000.

Stacey KJ, Fowles LF, Colman MS, Ostrowski MC, Hume DA. 1995. Regulation of urokinase-type plasminogen activator gene transcription by macrophage colony-stimulating factor. Mol Cell Biol 15: 3430-3441.

Taylor BS, Schultz N, Hieronymus H, Gopalan A, Xiao Y, Carver BS, Arora VK, Kaushik P, Cerami E, Reva B, et al. 2010. Integrative genomic profiling of human prostate cancer. Cancer Cell 18: 11-22.

Tomlins SA, Rhodes DR, Perner S, Dhanasekaran SM, Mehra R, Sun XW, Varambally S, Cao X, Tchinda J, Kuefer R, et al.
2005. Recurrent fusion of TMPRSS2 and ETS transcription factor genes in prostate cancer. Science 310: 644-648.

Tomlins SA, Mehra R, Rhodes DR, Smith LR, Roulston D, Helgeson BE, Cao X, Wei JT, Rubin MA, Shah RB, et al. 2006. TMPRSS2:ETV4 gene fusions define a third molecular subtype of prostate cancer. Cancer Res 66: 3396-3400.

Tomlins SA, Laxman B, Dhanasekaran SM, Helgeson BE, Cao X, Morris DS, Menon A, Jing X, Cao Q, Han B, et al. 2007. Distinct classes of chromosomal rearrangements create oncogenic ETS gene fusions in prostate cancer. Nature 448: 595-599.

Tomlins SA, Laxman B, Varambally S, Cao X, Yu J, Helgeson BE, Cao Q, Prensner JR, Rubin MA, Shah RB, et al. 2008. Role of the TMPRSS2-ERG gene fusion in prostate cancer. Neoplasia 10: $177-188$.

Turner DP, Findlay VJ, Moussa O, Semenchenko VI, Watson PM, Larue AC, Desouki MM, Fraig M, Watson DK. 2011. Mechanisms and functional consequences of PDEF protein expression loss during prostate cancer progression. Prostate. doi: $10.1002 /$ pros.21389.

Verger A, Buisine E, Carrere S, Wintjens R, Flourens A, Coll J, Stehelin D, Duterque-Coquillaud M. 2001. Identification of amino acid residues in the ETS transcription factor Erg that mediate Erg-Jun/Fos-DNA ternary complex formation. J Biol Chem 276: 17181-17189.

Vitari AC, Leong KG, Newton K, Yee C, O'Rourke K, Liu J, Phu L, Vij R, Ferrando R, Couto SS, et al. 2011. COP1 is a tumour suppressor that causes degradation of ETS transcription factors. Nature 474: 403-406.

Wasylyk B, Hagman J, Gutierrez-Hartmann A. 1998. Ets transcription factors: nuclear effectors of the Ras-MAP-kinase signaling pathway. Trends Biochem Sci 23: 213-216.

Weber MJ, Gioeli D. 2004. Ras signaling in prostate cancer progression. J Cell Biochem 91: 13-25.

Wei GH, Badis G, Berger MF, Kivioja T, Palin K, Enge M, Bonke M, Jolma A, Varjosalo M, Gehrke AR, et al. 2010. Genomewide analysis of ETS-family DNA-binding in vitro and in vivo. $E M B O$ I 29: 2147-2160.

Wu J, Janknecht R. 2002. Regulation of the ETS transcription factor ER81 by the 90-kDa ribosomal S6 kinase 1 and protein kinase A. J Biol Chem 277: 42669-42679.

Yordy JS, Muise-Helmericks RC. 2000. Signal transduction and the Ets family of transcription factors. Oncogene 19: 65036513.

Yu J, Mani RS, Cao Q, Brenner CJ, Cao X, Wang X, Wu L, Li J, Hu M, Gong Y, et al. 2010. An integrated network of androgen receptor, polycomb, and TMPRSS2-ERG gene fusions in prostate cancer progression. Cancer Cell 17: 443-454.

Zeeberg BR, Feng W, Wang G, Wang MD, Fojo AT, Sunshine M, Narasimhan S, Kane DW, Reinhold WC, Lababidi S, et al. 2003. GoMiner: a resource for biological interpretation of genomic and proteomic data. Genome Biol 4: R28. doi: 10.1186/gb-2003-4-4-r28.

Zong Y, Xin L, Goldstein AS, Lawson DA, Teitell MA, Witte ON. 2009. ETS family transcription factors collaborate with alternative signaling pathways to induce carcinoma from adult murine prostate cells. Proc Natl Acad Sci 106: 1246512470. 


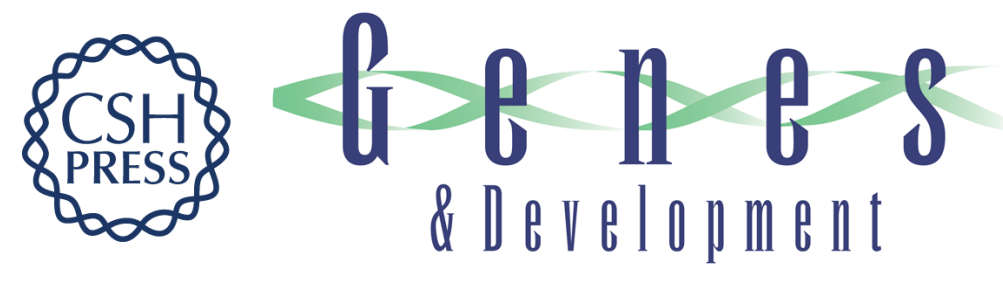

\section{Oncogenic ETS proteins mimic activated RAS/MAPK signaling in prostate cells}

Peter C. Hollenhorst, Mary W. Ferris, Megan A. Hull, et al.

Genes Dev. 2011, 25:

Access the most recent version at doi:10.1101/gad.17546311

\section{Supplemental http://genesdev.cshlp.org/content/suppl/2011/10/19/25.20.2147.DC1 Material}

References This article cites 54 articles, 18 of which can be accessed free at: http://genesdev.cshlp.org/content/25/20/2147.full.html\#ref-list-1

\section{License}

Email Alerting

Receive free email alerts when new articles cite this article - sign up in the box at the top Service 